Pacific Journal of Mathematic 


\title{
ON MATRICES ASSOCIATED WITH GENERALIZED INTERPOLATION PROBLEMS
}

\author{
Douglas N. Clark
}

Two classical interpolation theorems, due to Carathéodory -Fejér and Nevanlinna-Pick, deal with classes of functions analytic in the unit disk which take certain prescribed values at finitely many points there. The theorems express certain extrema of these classes as eigenvalues of finite matrices. In this paper, there is given a generalization of this type of interpolation, which involves inner functions. It is seen that a certain theorem about Hankel matrices and projections of Toeplitz matrices generalizes both of the above interpolation theorems. The theorem also provides a generalization of some recent work of the author on meromorphic interpolation and a continuous analogue of a theorem on Toeplitz forms and interpolation. Finally, the theorem has some consequences in the theory of infinite Hankel matrices.

In 1957, Nehari [8] obtained the norm of the infinite Hankel matrix

$$
\mathscr{H}=\left(a_{j+k-1}\right) \quad\left(j, k=1,2, \cdots, \sum\left|a_{j}\right|^{2}<\infty\right)
$$

as the infimum of the expressions

$$
\left\|\sum_{-\infty}^{-1} a_{-j} e^{i j \varphi}+\sum_{0}^{\infty} b_{j} e^{i j \varphi}\right\|_{\infty}
$$

where $0 \leq \varphi \leq 2 \pi$ and $\left\{b_{j}\right\}$ runs over all square-summable sequences. Nehari's theorem can be considered as a generalization of a theorem of Carathéodory and Fejér: given $n+1$ complex numbers $c_{0}, c_{1}, \cdots, c_{n}$, let $S$ be the set of all functions $f(z)$ analytic in $|z|<1$ and with a power series of the form $f(z)=c_{0}+c_{1} z+\cdots+c_{n} z^{n}+z^{n+1}(\cdots)$. Then inf $\left\|f\left(e^{i \varphi}\right)\right\|_{\infty}^{2}$ for $f \in S$ is the norm of a finite matrix $\mathscr{A}$ which is unitarily equivalent to $\mathscr{H} \mathscr{H}^{*}$ (cf. [2]).

To make it clear why we want to call Nehari's theorem a generalized interpolation theorem, we adopt a definition similar to that of Sarason [11]:

DEFINITION 1.1. Let $f(z), f_{0}(z) \in H^{2}$ (Hardy space of the unit disk), $f_{0}$ an inner function (i.e., $\left|f_{0}\left(e^{i \varphi}\right)\right|=1$, a.e.). A function $W(z) \in H^{2}$ will be said to interpolate $f(z)$ at $f_{0}$ if there exists a function $g_{1}(z) \in H^{2}$ such that 


$$
f(z)=W(z)+g_{1}(z) f_{0}(z) \text {. }
$$

If $\mathscr{C}$ is given by (1.1), let us write $\mathscr{C}=\mathscr{C}\left(e^{i \varphi} F\right)$ for any function $F\left(e^{i \varphi}\right) \in L^{2}(0,2 \pi)$ satisfying

$$
a_{j}=\frac{1}{2 \pi} \int_{0}^{2 \pi} F\left(e^{i \varphi}\right) e^{i j \varphi} d \varphi, \quad j=1,2, \cdots .
$$

In this notation, if $f, f_{0}$ are as in Definition 1.1, the norm of $\mathscr{\mathscr { C }}\left(e^{i \varphi} \bar{f}_{0} f\right)$ is given, according to Nehari, by $\left\|\mathscr{K}\left(e^{i \varphi} \bar{f}_{0} f\right)\right\|=\inf$ $\left\|h\left(e^{i \varphi}\right)\right\|_{\infty}$ for $h$ running over the class of functions which interpolate $f$ at $f_{0}$.

In a recent paper, Sarason [11] showed how this type of theorem can also be made to include the classical Nevanlinna-Pick theorem (i.e., concerning Definition 1.1 when $f_{0}$ is a finite Blaschke product with distinct zeros).

Our main theorem (Theorem 2.1 below) can be considered as the right half-plane analogue of the theorems of Nehari and Sarason. Actually, it is a simple consequence of Nehari's theorem and of a generalization of [1, Corollary 2.1]. It seems to give a more direct approach to both the Carathéodory-Fejér and Nevanlinna-Pick theorems, and it allows us to write down the analogue of the NevanlinnaPick matrix for the case in which $f_{0}$ is a Blaschke product with multiple zeros (\$3) (i.e., the case in which we want to interpolate $f$ and certain of its derivatives at finitely many points).

With this approach, we are also able to generalize the interpolation theorems of [2] to this situation $(\S 4)$.

In $\S 5$, we consider certain integral operators of the Wiener-Hopf type and obtain a continuous analogue of Carathéodory-Fejér's theorem.

In $\S 6$, we apply our resuts to the theory of infinite Hankel matrices.

2. Main theorem. Let $\mathscr{P}$ be a linear operator on a Hilbert space $X$. Throughout this section, we make the following.

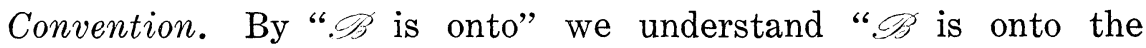
closure of its range, $\bar{R}(\mathscr{B})$." By $\mathscr{B}^{-1}$ we understand the generalized inverse of $\mathscr{B}$, i.e., $\mathscr{B}^{-1} x=0$ if $x \perp R(\mathscr{B})$ and if $x \in R(\mathscr{B}), \mathscr{B}^{-1} x$ is the (unique) element $y$ in the pre-image of $x$ such that $y \perp N(\mathscr{B})$, the nullspace of $\mathscr{B}$.

For a function $f\left(e^{i \circ}\right) \in L^{2}(0,2 \pi)$, we define the Laurent operator $\mathscr{L}(f)$ on $L^{2}$ by $\mathscr{L}(f) x=f x$, on the set of $x \in L^{2}$ for which $x f \in L^{2}$. If also $f \in H^{2}$, we define the analytic Toeplitz operator $\mathscr{T}(f)$ as the 
restriction of $\mathscr{L}(f)$ to $H^{2}$, i.e., the operator $\mathscr{T}$ with domain $D(\mathscr{T})$ $=\left\{x \in H^{2}: x f \in H^{2}\right\}$ and satisfying $\mathscr{T} x=f x$ for $x \in D(\mathscr{T})$.

The action of the matrix $\mathscr{H}\left(e^{i \varphi} F\right)\left(F \in L^{2}\right)$ on $H^{2}$ (with respect to the basis $1, e^{i \varphi}, e^{2 i \varphi}, \cdots$ of $H^{2}$, can be described as follows: For $x \in H^{2}$, the function $x F$ belongs to $L^{1}$ and has a Fourier series $x F \sim$ $\sum_{-\infty}^{\infty} x_{n} e^{i n \varphi}$. Now $x \in D\left(\mathscr{C}\left(e^{i \varphi} F\right)\right)$ whenever $y\left(e^{i \varphi}\right)=\sum_{-\infty}^{-1} x_{n} e^{i n \varphi} \in L^{2}$, in which case $\mathscr{H}\left(e^{i \varphi} F\right) x=e^{-i \varphi} y\left(e^{-i \varphi}\right) \in H^{2}$.

For $F\left(e^{i \varphi}\right) \in L^{2}$, we will use the operator $\mathscr{C}(F)$ defined by

$$
\mathscr{C}\left(e^{i \varphi} F\right)=\mathscr{H}\left(e^{i \varphi} F\right) \mathscr{H}\left(e^{i \varphi} F\right)^{*} .
$$

Furthermore, the operator $\mathscr{C}_{-}$is defined on $L^{2}$ by

$$
\mathscr{U} \_x(\varphi)=x(-\phi) \text {. }
$$

If $f_{0} \in H^{2}$ is an inner function, the subspace of all $H^{2}$ functions of the form $f_{0}(z) x(z), x \in H^{2}$, will be denoted $f_{0} H^{2}$.

Finally, for $x(\varphi) \in L^{2}, x^{*}(\varphi)$ will denote the function $\bar{x}(-\phi)$.

We need the following generalization of [1, Corollary 2.1].

LEMMA 2.1. Let $f_{0}(z) \in H^{2}$ be an inner function. Let $f(z), g(z)$ be $H^{2}$ functions, at least one of which belongs $H^{\infty}$. Then

$$
\mathscr{H}\left(e^{i \varphi} \bar{f}_{0} f g\right) \supset \mathscr{H}\left(e^{i \varphi} \bar{f}_{0} f\right) \mathscr{C} \mathscr{H}\left(e^{i \varphi} \cdot \bar{f}_{0} g\right)
$$

where

$$
\mathscr{Q} x=\mathscr{H}\left(e^{i \varphi} f_{0}\left(e^{-i \varphi}\right)\right) x=\mathscr{L}\left(e^{-i \varphi} f_{0}\right) \mathscr{L}_{-} x, x \in H^{2} .
$$

Actually, for the remainder of this section, we will be assuming both $f$ and $g$ belong to $H^{\infty}$. The full force of Lemma 2.1, however, will be used in $\S 6$.

Proof. For

$$
x\left(e^{i \varphi}\right) \in D\left(\mathscr{H}\left(e^{i \varphi} \bar{f}_{0} f\right) \mathscr{U} \mathscr{H}\left(e^{i \varphi} \bar{f}_{0} g\right)\right),
$$

let $y\left(e^{i \varphi}\right)=\mathscr{C}\left(e^{i \varphi} \bar{f}_{0} g\right) x$. Thus

$$
\begin{aligned}
e^{i \varphi} \bar{f}_{0}\left(e^{i \varphi}\right) g\left(e^{i \varphi}\right) x\left(e^{i \varphi}\right) & =y\left(e^{-i \varphi}\right)+e^{i \varphi} y_{1}\left(e^{i \varphi}\right), y_{1} \in H^{1} \\
& =\mathscr{U} \_y\left(e^{i \varphi}\right)+e^{i \varphi} y_{1}\left(e^{i \varphi}\right) . \\
g\left(e^{i \varphi}\right) x\left(e^{i \varphi}\right) & =\mathscr{L}\left(e^{-i \varphi} f_{0}\right) \mathscr{C}-y\left(e^{i \varphi}\right)+f_{0}\left(e^{i \varphi}\right) y_{1}\left(e^{i \varphi}\right) \\
e^{i \varphi} \bar{f}_{0} f g x & =e^{i \varphi} . \bar{f}_{0} f \mathscr{C} y+e^{i \varphi} y_{1} f
\end{aligned}
$$

where either $y_{1} \in H^{2}$ (if $g \in H^{\infty}$ ) or $f \in H^{\infty}$. Thus, in either case, $e^{i \varphi} y_{1} f \in H^{1}$, so that

$$
\mathscr{H}\left(e^{i \varphi} \bar{f}_{0} f g\right) x=\mathscr{H}\left(e^{i \varphi} \bar{f}_{0} f\right) \mathscr{U} y
$$


which proves $(2.1)$.

Lemma 2.2. Let $f_{0}, F, G, W \in H^{\infty}, f_{0}$ inner. Let $G \cdot W$ interpolate $F$ at $f_{0}$. Suppose further that $\mathscr{H}\left(e^{i \varphi} \bar{f}_{0} G\right)^{-1}$ is bounded. Then

$$
\begin{aligned}
& \mathscr{H}\left(\mathscr{I}-\mathscr{C}\left(e^{i \varphi} \bar{f}_{0} W\right)\right) \mathscr{C}^{*} \\
& =\mathscr{H}\left(e^{i \varphi} \bar{f}_{0} G\right)^{-1}\left[\mathscr{C}\left(e^{i \varphi} \bar{f}_{0} G\right)-\mathscr{C}\left(e^{i \varphi} \bar{f}_{0} F\right)\right] \mathscr{H}\left(e^{i \varphi} \bar{f}_{0} G\right)^{-1 *} .
\end{aligned}
$$

In particular, if $F=1-J, G=1+J$, where $|1+J| \geq c>0$ in $|z|<1$, then $W(z)=F / G \in H^{\infty}, \mathscr{H}\left(e^{i \varphi} \bar{f}_{0} G\right)^{-1}$ is bounded and

$$
\begin{aligned}
& \mathscr{U}\left(\mathscr{I}-\mathscr{C}\left(e^{i \varphi} \bar{f}_{0} W\right)\right) \mathscr{C}^{*} \\
= & 2 \mathscr{C}\left(e^{i \varphi} \bar{f}_{0} G\right)^{-1} \mathscr{P}\left(\mathscr{T}\left(J^{*}\right)+\mathscr{T}\left(J^{*}\right)^{*}\right) \mathscr{P} \mathscr{C}\left(e^{i \varphi} \bar{f}_{0} G\right)^{-1 *}
\end{aligned}
$$

on $K=\left(f_{0}^{*} H^{2}\right)^{\perp}$, where $\mathscr{P}$ is the orthogonal projection onto $K$.

For more about the invertibility of $\mathscr{H}\left(e^{i \varphi} \bar{f}_{0} G\right)$, see $\S 6$ below.

Proof. Since $G W$ interpolates $F$ at $f_{0}$, we have $F=W G+f_{0} g_{1}$. Thus, by Lemma 2.1, $\mathscr{\mathscr { C }}\left(e^{i \varphi} \bar{f}_{0} F\right) \supset \mathscr{\mathscr { C }}\left(e^{i \varphi} \bar{f}_{0} G\right) \mathscr{\mathscr { C }} \mathscr{\mathscr { C }}\left(e^{i \varphi} \bar{f}_{0} W\right)$ and the two are equal, since the right side is bounded. Now, since $R(\mathscr{U})=$ $\left(f_{0} H^{2}\right)^{\perp}=N\left(\mathscr{H}\left(e^{i \varphi} \bar{f}_{0} G\right)\right)^{\perp}$, we see that $\mathscr{H}\left(e^{i \varphi} \bar{f}_{0} G\right)$ invertible implies

$$
\mathscr{H}\left(e^{i \varphi} \bar{f}_{0} G\right)^{-1} \mathscr{H}\left(e^{i \varphi} \bar{f}_{0} F\right)=\mathscr{C} \mathscr{H}\left(e^{i \varphi} T_{0} W\right) .
$$

Taking adjoints,

$$
\mathscr{H}\left(e^{i \varphi} \bar{f}_{0} F\right)^{*} \mathscr{H}\left(e^{i \varphi} \bar{f}_{0} G\right)^{-1 *}=\mathscr{H}\left(e^{i \varphi} \bar{f}_{0} W\right)^{*} \mathscr{U}^{*} .
$$

Multiplying the last two relations gives

$$
\mathscr{H}\left(e^{i \varphi} \bar{f}_{0} G\right)^{-1} \mathscr{C}\left(e^{i \varphi} \bar{f}_{0} F\right) \mathscr{H}\left(e^{i \varphi} \bar{f}_{0} G\right)^{-1 *}=\mathscr{U} \mathscr{C}\left(e^{i \varphi} \bar{f}_{0} W\right) \mathscr{U}^{*} .
$$

But now it is easy to see that

$$
\mathscr{U} \mathscr{U}^{*}=\mathscr{H}\left(e^{i \varphi} \bar{f}_{0} G\right)^{-1} \mathscr{C}\left(e^{i \varphi} \bar{f}_{0} G\right) \mathscr{H}\left(e^{i \varphi} \bar{f}_{0} G\right)^{-1} .
$$

Adding this to (2.4) gives (2.1).

In case $F=1-J, G=1+J$, it is easily seen from Lemma 2.1 applied with $g=1+J, f=(1+J)^{-1}$ that $\mathscr{H}\left(e^{i \varphi} \bar{f}_{0} G\right)$ is invertible. $\mathscr{C}\left(e^{i \varphi} \bar{f}_{0}(1-J)\right)-\mathscr{C}\left(e^{i \varphi} \bar{f}_{0}(1+J)\right)=2\left(\mathscr{T}\left(J^{*}\right)+\mathscr{T}\left(J^{*}\right)^{*}\right)$ follows easily from the characterization $\mathscr{T}\left(J^{*}\right)^{*} x=\mathscr{P}_{+} \bar{J}^{*} x$, where $x \in H^{2}$ and $\mathscr{P}_{+}$is the orthogonal projection from $L^{2}$ to $H^{2}$ (cf. [2]).

Now we can state our main result.

THEOREM 2.1. Let $f(z), f_{0}(z) \in H^{\infty}, f_{0}$ an inner function. Let $S$ be the set of $H^{2}$ functions which interpolate $f$ at $f_{0}$. Let $\mathscr{P}$ be the orthogonal projestion on $K=\left(f_{0}^{*} H^{2}\right)^{\perp}$. Then 


$$
\mu_{0}=\sup _{h \in S} \inf _{|z|<1} \operatorname{Re} h(z)
$$

is the smallest point of the spectrum of

$$
\mathscr{T}_{K}(f)=\left.\frac{1}{2} \mathscr{P}\left[\mathscr{T}\left(f^{*}\right)+\mathscr{T}\left(f^{*}\right)^{*}\right]\right|_{K}
$$

the restriction of $\mathscr{P}\left[\mathscr{T}\left(f^{*}\right)+\mathscr{T}\left(f^{*}\right)^{*}\right]$ to $K$.

REMARK. The classical interpolation theorems mentioned above, do not include the requirement $S \subset H^{2}$. This requirement is necessary if we are to consider interpolation with respect to an inner function which is not a Blaschke product. In case $f_{0}$ is a Blaschke product, however, the requirement may more conveniently be dropped (cf. Corollary 3.1).

Proof. Let $\varepsilon \geq 0$ and suppose $S$ contains an $h(z) \in H^{2}$ with $\operatorname{Re} h(z) \geq \mu_{0}-\varepsilon$ in $|z|<1$. Let $h_{\varepsilon}(z)=h(z)-\mu_{0}+\varepsilon$. Then

and

$$
g_{\varepsilon}(z)=\left(1-h_{\varepsilon}(z)\right) /\left(1+h_{\varepsilon}(z)\right) \in H^{\infty},
$$

$$
\left\|\mathscr{H}\left(e^{i \varphi} g_{\varepsilon}\left(e^{i \varphi}\right) \bar{f}_{0}\left(e^{i \varphi}\right)\right)\right\| \leq 1
$$

by Nehari [8]. Now we can apply Lemma 2.2 with $W(z)=g_{\varepsilon}(z)$ and $J(z)=h_{\varepsilon}(z)$, and obtain

$$
\mathscr{J}-\mathscr{C}\left(e^{i \varphi} \bar{f}_{0} g_{\varepsilon}\right)=2 \mathscr{U}^{*} \mathscr{B} \mathscr{P}\left(\mathscr{T}\left(h_{\varepsilon}{ }^{*}\right)+\mathscr{T}\left(h_{\varepsilon}{ }^{*}\right)^{*}\right) \mathscr{P} \mathscr{B} * \mathscr{U}
$$

where $\mathscr{B}=\mathscr{C}\left(e^{i \varphi} \bar{f}_{0}\left(1-h_{\varepsilon}\right)\right)^{-1}$. Thus (2.5) implies $\left\|\mathscr{C}\left(e^{i \varphi} \bar{f}_{0} g_{\varepsilon}\right)\right\| \leq 1$, so that the right side of (2.6) is a nonnegative operator. It is easy to see that this implies

$$
0 \leq \frac{1}{2} \mathscr{T}_{K}\left(h_{\varepsilon}\right)=\frac{1}{2}\left(\mathscr{T}_{K}(h)-\left(\mu_{0}-\varepsilon\right) \mathscr{J}\right) .
$$

Thus, if we note that $h$ interpolating $f$ at $f_{0}$ implies $\mathscr{T}_{K}(h)=\mathscr{T}_{K}(f)$, we see that

$$
\frac{1}{2} \mathscr{T}_{K}(f) \geq \mu_{0}
$$

To complete the proof of the theorem. Choose an $h \in S$ with $\operatorname{Re} h(z) \geq \mu_{0}-\delta$ in $|z|<1$, for some $0 \leq \delta<1$. It is easily seen that (2.6) holds with $\varepsilon=0$ and that all operators involved are bounded. Now if the strict inequality holds in (2.7), we see from (2.6) with $\varepsilon=0$ that $\left\|\mathscr{C}\left(e^{i \varphi} g_{0} \bar{f}_{0}\right)\right\|<1$ and by Nehari's Theorem there is a $g(z) \in H^{\infty},\left|g\left(e^{i \varphi}\right)\right|<C<1$ and $g$ interpolates $g_{0}$ at $f_{0}$. Let

$$
g_{1}=(1-g)(1+g)^{-1} \in H^{\infty} \text {. }
$$


Notice that

$g_{1}-h_{0}=(1-g)(1+g)^{-1}-\left(1-g_{0}\right)\left(1+g_{0}\right)^{-1}=\left(g_{0}-g\right)\left[(1+g)\left(1+g_{0}\right)\right]^{-1}$

so $g_{1}$ interpolates $h_{0}$ (and hence $f-\mu_{0}$ ) at $f_{0}$. But $\operatorname{Re} g_{1} \geqq c^{\prime}>0$ which contradicts the definition of $\mu_{0}$.

3. The classical interpolation theorems. In this section, we deal with the instance of Theorem 2.1 in which $f_{0}$ is a finite Blaschke product. First, we make a simple observation.

Corollary 3.1. In case $f_{0}$ is a Blaschke product (finite or infinite) Theorem 2.1 is correct if the class $S$ is replaced by the class of functions analytic in $|z|<1$ which vanish at the zeros of $f_{0}$.

The proof is clear from that of Theorem 2.1.

Now we turn to the case in which $f_{0}$ is a finite Blaschke product. We will consider separately the cases in which (a) $f_{0}(z)=z^{n+1}$, (b) $f_{0}(z)$ has distinct zeros and (c) the general case.

(a) Theorem 2.1 (i.e., Corollary 3.1) with $f_{0}(z)=z^{n+1}$ is precisely the classical Carathéodory-Fejér theorem in the following form:

THEorem 3.1. (Carathéodory-Fejér). Given $n+1$ constants $c_{0}$, $c_{1}, \cdots, c_{n}, c_{0}$ real, let $S$ be the set of all functions $f(z)$ analytic in $|z|<1$ and with a power series of the form $f(z)=c_{0}+2 c_{1} z+\cdots$ $+2 c_{n} z^{n}+z^{n+1}(\cdots)$. Then $\sup _{f \in S} \inf _{|z|<1} \operatorname{Re} f(z)$ is the smallest eigenvalue of the Toeplitz matrix $\left(c_{j-k}\right), j, k=0, \cdots, n, c_{-k}=\bar{c}_{k}$.

See [11] for references.

(b) Let $f_{0}(z)=\prod_{\nu=1}^{n}\left(z-\alpha_{\nu}\right) /\left(1-\bar{\alpha}_{\nu} z\right), \alpha_{\nu}$ distinct. In this case, $K=\left(f_{0}^{*} H^{2}\right)^{\perp}$ is spanned by the functions $p_{j}(z)=\left(1-\alpha_{j} z\right)^{-1}$. These functions do not form an orthonormal basis for $K$, but the matrix for $\mathscr{T}_{K}(f)$ is nonnegative definite if and only if the matrix $\left(c_{j k}\right)=$ $\left(\left(\mathscr{T}_{K}(f) p_{j}, p_{k}\right)\right)$ is. An easy computation yields

$$
\begin{aligned}
c_{j k} & =\left(\mathscr{T}\left(f^{*}\right) p_{j}, p_{k}\right)+\left(p_{j}, \mathscr{T}\left(f^{*}\right) p_{k}\right) \\
& =\left(f^{*} p_{j}, p_{k}\right)+\left(p_{j}, f^{*} p_{k}\right) \\
& =f^{*}\left(\bar{\alpha}_{k}\right)\left(1-\alpha_{j} \bar{\alpha}_{k}\right)^{-1}+\bar{f}^{*}\left(\bar{\alpha}_{j}\right)\left(1-\alpha_{j} \bar{\alpha}_{k}\right)^{-1} \\
& =\left(\bar{f}\left(\alpha_{k}\right)+f\left(\alpha_{j}\right)\right) /\left(1-\alpha_{j} \bar{\alpha}_{k}\right)
\end{aligned}
$$

and we have 
THEOREM 3.2. (Nevanlinna-Pick). Let $S$ be the class of functions analytic in $|z|<1$ which interpolate $f(z)$ at $f_{0}$. Then $S$ contains a $g(z)$ with $\operatorname{Re} g(z) \geqq 0,|z|<1$, if and only if the matrix $\left(c_{j k}\right)$ is nonnegative.

See [11] for references.

(c) In the general case, we take as basis for $\left(f_{0}^{*} H^{2}\right)^{\perp}$ the set of elements $p_{j 1}(z)=\left(1-\alpha_{j} z\right)^{-1}, \quad p_{j 2}(z)=\left(z-\bar{\alpha}_{j}\right) /\left(1-\alpha_{j} z\right)^{2}, \cdots, p_{j a}(z)=$ $\left(z-\bar{\alpha}_{j}\right)^{a-1} /\left(1-\alpha_{j} z\right)^{a}$, where $j=1, \cdots, n$ and where $a=a(j)$ is the multiplicity of $\alpha_{j}$ as a zero of $f_{0}(z)$. A computation similar to the above, only using Cauchy's formula for the derivatives of an analytic function gives

$$
\begin{aligned}
\left(\mathscr{T}_{K}(f) p_{j a}, p_{k b}\right)= & \left.\frac{1}{(b-1) !} \frac{d^{b-1}}{d z^{b-1}}\left(\frac{\left(z-\alpha_{j}\right)^{a-1}}{\left(1-\bar{\alpha}_{j} z\right)^{a}}\left(1-\bar{\alpha}_{k} z\right)^{b-1} f(z)\right)\right|_{\alpha_{k}} ^{-} \\
& +\left.\frac{1}{(a-1) !} \frac{d^{a-1}}{d z^{a-1}}\left(\frac{\left(z-\alpha_{k}\right)^{b-1}}{\left(1-\bar{\alpha}_{k} z\right)^{b}}\left(1-\bar{\alpha}_{j} z\right)^{a-1} f(z)\right)\right|_{\alpha_{j}}
\end{aligned}
$$

where $A^{-}$denotes $\bar{A}$.

4. Interpolation by meromorphic functions. In this section, we indicate how analogues of the results of [2] may be obtained for the operators $\mathscr{T}_{K}(f)$.

We need a generalization of Definition 1.1.

DEFINITION 4.1. Let $f(z), f_{1}(z), f_{2}(z), f_{0}(z) \in H^{2}, f_{0}$ inner. We will say that the function $W(z)=f_{1}(z) / f_{2}(z)$ interpolates $f(z)$ at $f_{0}$, provided

$$
f(z)=W(z)+g_{1}(z)
$$

for $|z|<1$ such that $f_{2}(z) \neq 0$, where $g_{1}(z)$ is a meromorphic function in $|z|<1$ which satisfies $f_{2}(z) g_{1}(z) \in f_{0} H^{1}$.

Let $f(z), f_{0}(z) \in H^{2}, f_{0}$ inner. We define the class $S_{k}\left(f_{0}, f\right)=S_{k}(f)$ as the set of functions $w(z)=f_{1}(z) / f_{2}(z), f_{2}(z)=\left(z-\alpha_{1}\right) \cdots\left(z-\alpha_{k}\right)$, $\left|\alpha_{j}\right|<1$, which interpolate $f(z)$ at $f_{0}(z) . S_{k}^{\gamma}\left(f_{0}, f\right)$ will denote the set of $G(z) \in S_{k}\left(f_{0}, f\right)$ such that the image of $|z|=1$ under $G(z)$ is contained in one of the disks $D_{\zeta, \delta}=\{|z-\zeta| \leqq \delta\}$, for some real $\zeta, \delta<\gamma$. Thus the class $S_{k}\left(f_{0}, f\right)$, for example, is the set of $w(z)$ of the form

$$
w(z)=f(z)+f_{0}(z) g(z)\left[\left(z-\alpha_{1}\right) \cdots\left(z-\alpha_{k}\right)\right]^{-1}
$$

where $g \in H^{2},\left|\alpha_{j}\right|<1, j=1,2, \cdots, k$.

We will also need the notion of the enumeration of the lower part of the spectrum sp $\mathscr{T}$ of an operator $\mathscr{T}$ on a Hilbert space $X$. 
Suppose $\mathscr{T}$ is self adjoint and bounded below. In case $\operatorname{dim} X=\infty$, let $\mu_{e}(\mathscr{T})$ denote the smallest real number which is either a cluster point of sp $\mathscr{T}$ or an eigenvalue of $\mathscr{T}$ of infinite multiplicity. The enumeration of the lower part of $\mathrm{sp} \mathscr{T}$ is then to mean the sequence

(a) $\mu_{0}, \mu_{1}, \cdots, \mu_{n}$ (where $\left.\operatorname{sp} \mathscr{T}=\left\{\mu_{0} \leqq \mu_{1} \leqq \cdots \leqq \mu_{n}\right\}\right)$ in case $n+1=\operatorname{dim} X<\infty$.

(b) $\mu_{0}, \mu_{1}, \cdots, \mu_{k-1}, \mu_{e}(\mathscr{T}), \mu_{e}(\mathscr{T}), \cdots$ in case there are $k$ points

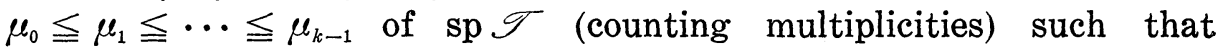
$\mu_{j}<\mu_{e}(\mathscr{T})$ (where $\left.0 \leqq k<\infty\right)$.

(c) $\mu_{0}, \mu_{1}, \cdots$ in case there are infinitely many points $\mu_{0} \leqq \mu_{1} \leqq$ (including multiplicities) in sp $\mathscr{T}$ satisfying $\mu_{j}<\mu_{e}(\mathscr{T})$.

Now we can state

THEOREM 4.1. Let $S_{j}^{\prime}=S_{j}^{\gamma}\left(f_{0}, f\right)$ for $\gamma$ a positive real number. Let $\mu_{0}, \mu_{1}, \cdots$ be an enumeration of the lower part of $\operatorname{sp} \mathscr{T}_{K}(f)$, where the Hilbert space $X=K$. Then

$$
\mu_{j}=\sup _{G \in S_{j}^{\prime}} \sup (\zeta-\delta)-(1-\mu)(1+\mu)^{-1}
$$

where $\mu=\gamma^{-1}\left(\sqrt{1+\gamma^{2}}-1\right)$ and where the second supremum is taken over all circles $D_{\zeta, \delta}(\zeta, \delta$ real, $0<\delta<\gamma)$ which contain the image under $G$ of $|z|=1$.

The theorem is a straightforward generalization of the case $f_{0}(z)$ $=z^{n+1}$ (cf. [2]) and its proof will only be sketched here. It is also an easy matter to write down the analogue of Theorem 2(b) of [2] for the present situation. This will be left to the reader.

Sketch of proof. The first step of the proof is to obtain the analogue of Theorem 2(a) of [2] for $S_{k}^{\gamma}\left(f_{0}, f\right)$. This follows easily from Theorem 2(a) of [2] and from the considerations of $\S 4$ of [1].

The next step is to let $\lambda=\sup \sup (\zeta-\delta)$ for $D_{\zeta, \delta} \supset h(|z|=1)$, $h \in S_{k i}^{\gamma}\left(f_{0}, f\right)$, and consider

$$
g(z)=(1-\mu(h-\eta))(1+\mu(h-\eta))^{-1}
$$

where $\eta=\lambda-(1-\mu)(1+\mu)^{-1}-\varepsilon$. As in [2], one can prove that $g(z)$ has $k$ poles in $|z|<1$ and $|g(z)|$ is bounded (by 1) in $1-\delta<$ $|z| \leqq 1$, for sufficiently small $\delta>0$. Thus $g(z)$ has the form

$$
g(z)=g_{1}(z)+g_{2}(z)\left[\left(z-\alpha_{1}\right) \cdots\left(z-\alpha_{k}\right)\right]^{-1}
$$

where $g_{1}, g_{2} \in H^{\infty}$ and $\left|\alpha_{j}\right|<1$. Now observe that $g(z)$ is interpolated at $f_{0}(z)$ by the $H^{\infty}$ function

$$
G(z)=g_{1}(z)+g_{2}(z) \prod_{\nu=1}^{k}\left[1-f_{0}(z) f_{0}\left(\alpha_{\nu}\right)^{-1}\right]\left[\left(z-\alpha_{1}\right) \cdots\left(z-\alpha_{k}\right)\right]^{-1} \cdot
$$


Thus $g(z) \in S_{k}\left(f_{0}, G(z)\right)$. The inequality $\mu_{j} \geqq \lambda-(1-\mu)(1+\mu)^{-1}$ now follows by applying step one to the class $S_{k}(G(z))$. The proof of the reverse inequality follows the lines of the proof in [2] with modifications similar to the above.

5. Finite section Wiener-Hopf operators. In this section, we consider the case in which $f_{0}$ is the singular inner function given by

$$
f_{0}(z)=\exp r\left[(z-1)(z+1)^{-1}\right],
$$

$r$ a nonnegative real number. Throughout this section, we will write $H^{2}(D)$ for $H^{2}$ of $D=\{|z|<1\}$ (instead of just $H^{2}$ ) because it will be convenient to map $H^{2}(D)$ onto $H^{2}(E)(E=\{\operatorname{Im} z>0\})$ and take Fourier transforms. We will define the various unitary maps and state the result; details may be found, e.g., in Hoffman's book [7].

With this aim, let $C$ denote the unit circle and

$$
\mathscr{V}_{1}: L^{\infty}(C) \longrightarrow L^{\infty}(-\infty, \infty)
$$

the isometry defined by $\mathscr{\mathscr { T }}_{1} g\left(e^{i \varphi}\right)=g\left([x-i][x+i]^{-1}\right)$ and let

$$
\text { थै: } H^{2}(D) \longrightarrow H^{2}(E)
$$

be defined by $h(w)=\mathscr{V}_{2}(g(z))=\sqrt{2}(w+i)^{-1} \mathscr{V} g(z)$. Then $\mathscr{V}_{2}$ is a unitary operator. For $h(w) \in H^{2}(E)$ or, more generally, for $h(x) \in L^{2}(-\infty, \infty)$, the Fourier transform $\hat{h}$ is defined by

$$
\hat{h}(t)=\frac{1}{2 \pi} \int_{-\infty}^{\infty} e^{-i t x} h(x) d x .
$$

The map $h \rightarrow \hat{h}$ maps $L^{2}(-\infty, \infty)$ isomorphically onto $L^{2}(-\infty, \infty)$ and sends the subspace $H^{2}(E)$ onto $L^{2}(0, \infty)$. (Here we use the convention that, if $I$ is a real interval, $L^{2}(I)$ denotes the subspace of functions in $L^{2}(-\infty, \infty)$ which vanish off $I$.) Let

$$
\text { Y : } H^{2}(D) \longrightarrow L^{2}(0, \infty)
$$

denote the unitary map $\mathscr{Y} g(z)=\left(\mathscr{\mathscr { V }}_{2} g\right)^{\wedge}$.

The reason for presenting these operators is that, as is easily seen, if $\mathscr{T}(f)$ is an analytic Toeplitz operator on $H^{2}(D)$,

$$
\mathscr{W}(g)=\mathscr{Y}\left(\mathscr{T}\left(f^{*}\right)^{*}+\mathscr{T}\left(f^{*}\right)\right) \mathscr{Y}^{*}
$$

is the Wiener-Hopf operator

$$
\mathscr{W}(g) x(t)=\int_{0}^{\infty} g(s-t) x(s) d s=g^{*} x
$$

where $g(t)=\left[\mathscr{V}_{1}\left(f^{*}\left(e^{i \varphi}\right)+\bar{f}^{*}\left(e^{i \varphi}\right)\right)\right]^{\wedge}$. Furthermore, the operator $\mathscr{T}_{K}(f)$, 
where $K=\left(f_{0}^{*} H^{2}(D)\right)^{\perp}, f_{0}$ given by (5.1) also takes a recognizable form under $\mathscr{Y}$. In fact, if $\mathscr{P}$ is the projection of $H^{2}(D)$ onto $K$,

$$
Q^{P}=\mathscr{Y} \mathscr{P}^{*}
$$

is the projection of $L^{2}(0, \infty)$ onto $L^{2}(0, r)$, cf., Sarason [10, p. 246], and thus

$$
\begin{aligned}
\mathscr{Y} \mathscr{T}_{K}(f) \mathscr{Y}^{*} & =\left.\mathscr{Q}_{\mathscr{W}}(g)\right|_{L^{2}(0, r)} \\
& =\mathscr{W}_{r}(g),
\end{aligned}
$$

where $\mathscr{W}_{r}(g)$ is the "finite section" Wiener-Hopf operator on $L^{2}(0, r)$

$$
\mathscr{W}_{r}(g) x(t)=\int_{0}^{r} g(s-t) x(s) d s .
$$

The operator $\mathscr{W}_{r}(g)$ has been studied in some detail, cf. [6] and the bibliography there.

With this notation, we can state Theorem 2.1 for the case at hand.

Theorem 5.1. Let $g(t) \in L^{2}(-r, r), g(-t)=\bar{g}(t)$ and let

$$
\mu_{0}=\sup \inf \operatorname{Re} h(w) \quad \operatorname{Im} w>0
$$

where the supremum is taken over the set of functions $h(w)$ in $H^{2}(E)$ such that $\hat{h}$ agrees $g$ on $[0, r]$. Then $\mu_{0}$ is the smallest point of the spectrum of $\mathscr{W}_{r}(g)$.

In contemporary language, this theorem is the "continuous analogue" of the Carathéodory-Fejér Theorem 3.1.

From the above considerations and Theorem 4.1 above, one can immediately deduce the continuous analogues of Theorem 2(b) of [2]. This is straightforward and will not be included here.

It can be noted that, in the case of analytic kernels (i.e., $g(t)$ supported on $[0, r])$, the theorem of Nehari [8] has a very simple analogue for $\mathscr{W}_{r}(g)$. By an obvious generalization of Lemma 2.1 of [1], we see that, if $f \in H^{\infty}, \mathscr{C}\left(e^{i \varphi} \bar{f}_{0} f\right)=\left.\mathscr{U}^{*} \mathscr{P} \mathscr{T}\left(f^{*}\right)\right|_{K}, K=\left(f_{0}^{*} H^{2}(D)\right)^{\perp}$. In particular, $\left\|\mathscr{K}\left(e^{i \varphi} \bar{f}_{0} f\right)\right\|=\left\|\mathscr{T}^{K}(f)\right\|$, where $\mathscr{T}^{K}(f)=\left.\mathscr{P} \mathscr{T}(f)\right|_{K}$. It follows at once that

TheOREM 5.2. Let $g(t) \in L^{2}(0, r)$ and let

$$
\lambda_{0}=\inf \|h(w)\|_{\infty} \quad \operatorname{Im} w>0
$$

where the infimum is taken over the set of functions $h \in H^{2}(E)$ such that $\hat{h}$ agrees with $g$ on $(0, r)$. Then $\lambda_{0}$ is the norm of $\mathscr{W}_{r}(g)$. 
6. Invertibility of Hankel matrices. In the proof of Lemma 2.2, we used a consequence of Lemma 2.1 about the invertibility of $\mathscr{H}\left(e^{i \varphi} \bar{f}_{0} f\right)$. In this section, we will set down this invertibility criterion and investigate its consequences.

Before we proceed to the invertibility result, a few comments are in order. First, Hankel matrices are never invertible, with bounded inverses defined on all of $H^{2}$. In fact, 0 belongs to the essential spectrum of every bounded, Hermitian Hankel matrix; Hartman-Wintner [5, p. 366]. To extend this result to non-Hermitian and even unbounded Hankel matrices is a triviality. In fact, $\mathscr{H}$ cannot be invertible since if $\mathscr{H} x\left(e^{i \varphi}\right)=1$, it is easily seen that $\mathscr{C} e^{i \varphi} x\left(e^{i \varphi}\right)=0$. Similarly, $\mathscr{H} y\left(\mathrm{e}^{i \varphi}\right)=0$ implies that $\mathscr{H} e^{i n \varphi} y\left(e^{i \varphi}\right)=0, n=1,2, \ldots$ Thus for any Hankel matrix $\mathscr{C}, 0$ is either a cluster point of the spectrum or an eigenvalue of infinite multiplicity. For this reason, we need consider only Hankel matrices $\mathscr{H}$ with nontrivial nullspaces $N(\mathscr{H})$ and look for their inverses as operators on $N(\mathscr{H})^{\perp}$.

Suppose $\mathscr{H}(F), F \in L^{2}$ is a Hankel matrix with $N(\mathscr{H}(F)) \neq\{0\}$. As was noted above, $x\left(e^{i \varphi}\right) \in N(\mathscr{H}(F))$ implies $e^{i \varphi} x\left(e^{i \varphi}\right) \in N(\mathscr{H}(F))$ and therefore, by a well-known theorem of Beurling, there is an inner function $f_{0}\left(e^{i \varphi}\right) \in H^{2}$ such that

$$
N(\mathscr{C}(F))=f_{0} H^{2} .
$$

In particular, $F\left(e^{i \varphi}\right) f_{0}\left(e^{i \varphi}\right)=\sum_{1}^{\infty} y_{n} e^{i n \varphi}=e^{i \varphi} y\left(e^{i \varphi}\right), y \in H^{2}$, so that $F$ is of the form $F=e^{i \varphi} \bar{f}_{0} y\left(e^{i \varphi}\right)$. This implies $F^{*}\left(e^{i \varphi}\right)=e^{i \varphi} f_{0}\left(e^{-i \varphi}\right) y^{*}\left(e^{i \varphi}\right)$, so that $\bar{R} \mathscr{H}(F))=\left(f_{0}^{*} H^{2}\right)^{\perp}$. Thus the set of Hankel matrices we have been considering in earlier sections is the class of Hankel matrices having 0 as an eigenvalue. For various conditions on an $L^{2}$ function $F$ that $\mathscr{C}(F)$ should belong to this class, see Putnam [9, p. 657] and Douglas, Shapiro and Shields [3].

We now state a consequence of Lemma 2.1.

THEOREM 6.1. Let $f, W, f_{0} \in H^{2}, f_{0}$ inner. Let $W$ interpolate $1 / f$ at $f_{0}^{*}$ and suppose at least one of the functions, $f, W$ is in $H^{\infty}$. Then, on $K=\left(f_{0}^{*} H^{2}\right)^{\perp}$.

$$
\mathscr{H}\left(e^{i \varphi} \bar{f}_{0} f\right)^{-1}=\mathscr{U}^{*} \mathscr{H}\left(e^{i \varphi} f_{0}\left(e^{-i \varphi}\right) W\right) \mathscr{U} .
$$

Proof. The theorem follows easily from Lemma 2.1 with $g=W$, if one notices that in the three occurrences of $f_{0}$ on the right side of (2.1):

$$
\mathscr{H}\left(e^{i \varphi} \bar{f}_{0} f\right) \mathscr{L}\left(e^{-i \varphi} f_{0}\right) \mathscr{U}-\mathscr{H}\left(e^{i \varphi} \bar{f}_{0} W\right),
$$

$f_{0}$ could be replaced respectively by any three inner functions $g_{01}, g_{02}$, $g_{03}$ satisfying $\bar{f}_{0}=\bar{g}_{01} g_{02} \bar{g}_{03}$. 
From Sarason's Theorem 1 [11], we can obtain a variant of Theorem 6.1, which will be more useful for our purposes.

THEOREM 6.2. Let $f, f_{0} \in H^{2}, f_{0}$ inner and suppose that $\mathscr{H}\left(e^{i \varphi} \bar{f}_{0} f\right)$ has a bounded inverse on $\left(f_{0}^{*} H^{2}\right)^{\perp}$. Then there exists a $W \in H^{\infty}$ which satisfies (6.1).

Proof. If the functions $\psi, \phi$ are replaced by $f_{0}, W$ respectively, Theorem 1 of Sarason, loc. cit., states: a bounded operator $\mathscr{T}$ on $K^{\prime}=\left(f_{0} H^{2}\right)^{\perp}$ commutes with $\left.\mathscr{P}^{\prime} \mathscr{L}\left(e^{i \varphi}\right)\right|_{K}\left(\mathscr{P}^{\prime}\right.$ the projection onto $\left.K^{\prime}\right)$ if and only if there exists a $W \in H^{\infty}$ such that $\mathscr{T}=\mathscr{K} \mathscr{\mathscr { C }}\left(e^{i \varphi} \bar{f}_{0} W\right)$, $\mathscr{\mathscr { C }}$ is given in Lemma 2.1 above. Let $\mathscr{T}=\left(\mathscr{C} \mathscr{H}\left(e^{i \varphi} \bar{f}_{0} f\right)\right)^{-1}=$ $\mathscr{H}\left(e^{i \varphi} \bar{f}_{0} f\right)^{-1} \mathscr{C}^{*}$. By assumption, $\mathscr{T}$ is bounded and as is easily verified $\mathscr{T}$ commutes with $\left.\mathscr{P}^{\prime} \mathscr{L}\left(e^{i \varphi}\right)\right|_{K}{ }^{\prime}$. Thus

$$
\mathscr{H}\left(e^{i \varphi} \bar{f}_{0} f\right)^{-1}=\mathscr{U} \mathscr{H}\left(e^{i \varphi} \bar{f}_{0} W\right) \mathscr{U} .
$$

(6.1) follows by the observation made in the proof of Theorem 6.1.

From Theorem 6.2, we obtain

CoRollary 6.1. Let $F \in L^{2}$ and suppose $\mathscr{H}(F)$ is self-adjoint (not necessarily bounded), then 0 is an isolated point of $\operatorname{sp} \mathscr{H}(F)$ if and only if there exist functions $f, f_{0} \in H^{2}, f_{0}$ inner and $W \in H^{\infty}$ such that $F=e^{i \varphi} \bar{f}_{0} f$ and $W$ interpolates $1 / f$ at $f_{0}$. In this case (2.1) holds and $|\lambda|$, the smallest absolute value of a nonzero point of the spectrum of $\mathscr{H}(F)$ is given by

$$
|\lambda|=\left(\inf \|g\|_{\infty}\right)^{-1}
$$

where the infimum is taken over the set of $g \in H^{\infty}$ which interpolates $1 / f$ at $f_{0}$.

Proof. If 0 is an isolated eigenvalue, $\mathscr{H}(F)^{-1}$ is bounded on $\left(f_{0}^{*} H^{2}\right)^{\perp}$, so Theorem 6.2 applies. (6.2) follows from Nehari [8]. All the other statements are obvious.

The results of [1] may now be used to obtain a representation similar to (6.2) for the $j^{\text {th }}$ smallest absolute value of an eigenvalue of $\mathscr{C}(F)$. The details are easy and will not be given.

As a final application of Theorem 6.1, we give

CoRollary 6.2. Let $f, f_{0} \in H^{2}, f_{0}$ inner, $\mathscr{H}\left(e^{i \varphi} \bar{f}_{0} f\right)$ self adjoint. Then $\mathscr{Y}\left(e^{i \varphi} \bar{f}_{0} f\right)$ restricted to $\left(f_{0}^{*} H^{2}\right)^{\perp}$ has a completely continuous inverse if and only if $1 / f$ can be interpolated at $f_{0}$ by a function $W(z) \in H^{\infty}$ such that $\bar{f}_{0}\left(e^{i \varphi}\right) W\left(e^{i \varphi}\right)$ can be extended as a continuous, periodic function.

The corollary is a simple consequence of Corollary 6.1 and of a 
theorem of Hartman [4]. To construct examples of functions satisfying the conclusion of Corollary 6.2, it suffices to take an inner function $f_{0}$ whose support has measure zero (cf. [3]) and a function $f\left(e^{i \varphi}\right) \in H^{2}$ such that $1 / f(z)$ is continuous in the closed unit disk, analytic in its interior and $1 / f(z)=0$ on the support of $\bar{f}_{0}$. It follows that $\bar{f}_{0} / f$ is continuous since an inner function is continuous off its support.

REMARK. A sequence of points $z_{1}, z_{2}, \ldots$ in the unit disk is called an interpolating sequence if, for any bounded sequence $W_{1}, W_{2}$, ... of complex numbers, there is a function $g(z) \in H^{\infty}$ such that $g\left(z_{j}\right)$ $=W_{j}, j=1,2, \cdots$ (cf. [7, pp. $\left.\left.194 \mathrm{ff}.\right]\right)$. We hope to show in a separate paper that the assumption that $f_{0}(z)$ is a Blaschke product whose zeros form an interpolating sequence is a useful assumption when dealing with the Hankel matrices $\mathscr{C}\left(e^{i \varphi} \bar{f}_{0} f\right)$, and the Toeplitz matrices $\mathscr{T}_{K}(f)$.

\section{REFERENCES}

1. D. N. Clark, On the spectra of bounded, Hermitian, Hankel matrices, Amer. J. Math. 90 (1968), 627-656.

2. Hankel forms, Toeplitz forms and meromorphic functions, Trans. Amer. Math. Soc. 134 (1968), 109-116.

3. R. G. Douglas, H. S. Shapiro and A. L. Shields, On cyclic vectors of the backward shift, Bull. Amer. Math. Soc. 73 (1967), 156-159.

4. P. Hartman, On completely continuous Hankel matrices, Proc. Amer. Math. Soc. $\mathbf{9}$ (1958), 862-866.

5. P. Hartman and A. Wintner, On the spectra of Toeplitz's matrices, Amer. J. Math. 72 (1950), 359-366.

6. I. I. Hirschman, Jr., On a formula of Kac and Achiezer, J. Math. Mech. 16 (1966), 167-196.

7. K. Hoffman, Banach Spaces of Analytic Functions, Prentice-Hall, Inc., Englewood Cliffs, N. J., 1962.

8. Z. Nehari, On bounded bilinear forms, Ann. of Math. 65 (1957), 153-162.

9. C. R. Putnam, Toeplitz matrices and invertibility of Hankel matrices, Pacific J. Math. 14 (1964), 651-658.

10. D. Sarason, A remark on the Volterra operator, J. Math. Analysis and Applications 12 (1965), 244-246.

11. - Generalized interpolation in $H^{\infty}$, Trans. Amer. Math. Soc. 127 (1967), 179-203.

Received Dezember 11, 1967. This work was supported by an N. R. C. -O. N. R. Postdoctoral Research Associateship at the University of Wisconsin.

THE UNIVERSITY OF WISCONSIN

MADISON, WISCONSIN 



\section{PACIFIC JOURNAL OF MATHEMATICS}

\section{EDITORS}

\section{H. ROYDEN}

Stanford University

Stanford, California

\author{
R. R. Phelps \\ University of Washington \\ Seattle, Washington 98105
}

\section{J. DugundJI}

Department of Mathematics University of Southern California Los Angeles, California 90007

\section{RICHARD ARENS}

University of California

Los Angeles, California 90024

\section{ASSOCIATE EDITORS}

\section{E. F. BECKENBACH}

B. H. NEUMANN

F. WOLF

K. YOSIDA

\section{SUPPORTING INSTITUTIONS}

UNIVERSITY OF BRITISH COLUMBIA CALIFORNIA INSTITUTE OF TECHNOLOGY UNIVERSITY OF CALIFORNIA MONTANA STATE UNIVERSITY UNIVERSITY OF NEVADA NEW MEXICO STATE UNIVERSITY OREGON STATE UNIVERSITY UNIVERSITY OF OREGON OSAKA UNIVERSITY UNIVERSITY OF SOUTHERN CALIFORNIA

\author{
STANFORD UNIVERSITY \\ UNIVERSITY OF TOKYO \\ UNIVERSITY OF UTAH \\ WASHINGTON STATE UNIVERSITY \\ UNIVERSITY OF WASHINGTON \\ AMERICAN MATHEMATICAL SOCIETY \\ CHEVRON RESEARCH CORPORATION \\ TRW SYSTEMS
}

NAVAL WEAPONS CENTER

Mathematical papers intended for publication in the Pacific Journal of Mathematics should be in typed form or offset-reproduced, double spaced with large margins. Underline Greek letters in red, German in green, and script in blue. The first paragraph or two must be capable of being used separately as a synopsis of the entire paper. It should not contain references to the bibliography. Manuscripts, in duplicate if possible, may be sent to any one of the four editors. All other communications to the editors should be addressed to the managing editor, Richard Arens, University of California, Los Angeles, California 90024.

Each author of each article receives 50 reprints free of charge; additional copies may be obtained at cost in multiples of 50 .

The Pacific Journal of Mathematics is published monthly. Effective with Volume 16 the price per volume (3 numbers) is $\$ 8.00$; single issues, $\$ 3.00$. Special price for current issues to individual faculty members of supporting institutions and to individual members of the American Mathematical Society: $\$ 4.00$ per volume; single issues $\$ 1.50$. Back numbers are available.

Subscriptions, orders for back numbers, and changes of address should be sent to Pacific Journal of Mathematics, 103 Highland Boulevard, Berkeley 8, California.

Printed at Kokusai Bunken Insatsusha (International Academic Printing Co., Ltd.), 7-17, Fujimi 2-chome, Chiyoda-ku, Tokyo, Japan.

PUBLISHED BY PACIFIC JOURNAL OF MATHEMATICS, A NON-PROFIT CORPORATION

The Supporting Institutions listed above contribute to the cost of publication of this Journal, but they are not owners of publishers and have no responsibility for its content or policies. 


\section{Pacific Journal of Mathematics}

\section{Vol. 27, No. $2 \quad$ February, 1968}

Leonard E. Baum and George Roger Sell, Growth transformations for

functions on manifolds ............................ 211

Henry Gilbert Bray, A note on CLT groups ................... 229

Paul Robert Chernoff, Richard Anthony Rasala and William Charles

Waterhouse, The Stone-Weierstrass theorem for valuable fields....... 233

Douglas Napier Clark, On matrices associated with generalized

interpolation problems ................................

Richard Brian Darst and Euline Irwin Green, On a Radon-Nikodym theorem for finitely additive set functions . ...................... 255

Carl Louis DeVito, A note on Eberlein's theorem..................... 261

P. H. Doyle, III and John Gilbert Hocking, Proving that wild cells exist . . . 265

Leslie C. Glaser, Uncountably many almost polyhedral wild $(k-2)$-cells in

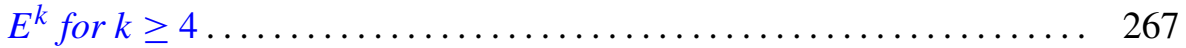

Samuel Irving Goldberg, Totally geodesic hypersurfaces of Kaehler

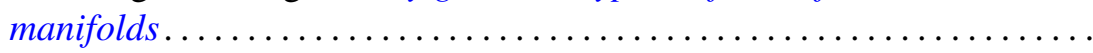

Donald Goldsmith, On the multiplicative properties of arithmetic functions .................................... 283

Jack D. Gray, Local analytic extensions of the resolvent ............ 305

Eugene Carlyle Johnsen, David Lewis Outcalt and Adil Mohamed Yaqub,

Commutativity theorems for nonassociative rings with a finite division ring homomorphic image ....................

André (Piotrowsky) De Korvin, Normal expectations in von Neumann

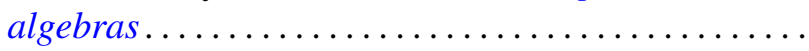

James Donald Kuelbs, A linear transformation theorem for analytic

Feynman integrals..........................

W. Kuich, Quasi-block-stochastic matrices ................... 353

Richard G. Levin, On commutative, nonpotent archimedean

semigroups ............................... 365

James R. McLaughlin, Functions represented by Rademacher series ... . . . 373

Calvin R. Putnam, Singular integrals and positive kernels............ 379

Harold G. Rutherford, II, Characterizing primes in some noncommutative

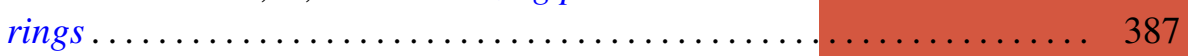

Benjamin L. Schwartz, On interchange graphs................... 393

Satish Shirali, On the Jordan structure of complex Banach *algebras . . . . . 397

Earl J. Taft, A counter-example to a fixed point conjecture............. 405

J. Roger Teller, On abelian pseudo lattice ordered groups ..... 